\title{
Torsional mode coupling and filtering in a composite waveguide with multiperiodic interface corrugations
}

\author{
Omar R. Asfar \\ Department of Electrical Engineering, University of Science and Technology, Irbid, Jordan \\ Muhammad A. Hawwa \\ Department of Engineering Science and Mechanics, Virginia Polytechnic Institute and State University, \\ Blacksburg, Virginia 24061
}

(Received 5 July 1992; accepted for publication 4 January 1993)

\begin{abstract}
This study is concerned with the interaction of six torsional modes in a composite axisymmetric waveguide whose interfaces are sinusoidally corrugated in the axial direction. The modes are interacting when two resonant conditions on the codirectional modes and a Bragg condition occur simultaneously. In light of the weakness of the interface corrugations, the perturbation method of multiple scales is used to derive the mode coupling equations. A novel numerical scheme for two-point boundary-value problems is used to solve the coupled amplitude equations. The power reflection coefficient of a filter section is then calculated for the cases of uniform, tapered, and chirped corrugations. An optimal filter is realized by combining both taper and chirp thus producing a nearly ideal characteristics.
\end{abstract}

PACS numbers: 43.20.Gp, 43.20.Mv, 43.40.Sk

\section{INTRODUCTION}

The interaction of modes of elastic waves propagating along a circular wire was observed experimentally by Meitzler. ${ }^{1}$ Hirao ${ }^{2}$ explained Meizler's experimental results analytically by considering the mode coupling of guided elastic waves in a wire with weak surface corrugation. It was shown that the amplitude variations characterize the pulse distortion observed in the experiments. Kim and $\mathrm{Bau}^{3}$ in their effort to model a real-time viscosimeter, focused on torsional stress waves in a circular cylinder with a modulated surface. They found that resonant conditions exist when the modulation wave numbers are proportional to the sum or difference of the wave numbers corresponding to various modes of the torsional stress wave. This result agrees with that obtained by Asfar and Nayfeh ${ }^{4}$ in their work on electromagnetic waves in a circular cylindrical waveguide with sinusoidally perturbed wall.

Mode coupling of elastic waves in a composite rod was first considered by Asfar and Nayfeh, ${ }^{5}$ who analyzed torsional waves in a clad rod having a sinusoidally perturbed core-cladding interface. They concluded that the mode coupling could be a desirable feature, if the periodic corrugations are built into the waveguide in an orderly manner and for specified length so as to produce energy transfer from one propagating mode into another.

In the present work, the general problem of torsional elastic waves in a clad rod with periodicities at both the interface and the outerface is considered. When dealing with signals at frequencies higher than cutoff of the dominant mode in such a waveguide, the existence of more modes requires a mechanism of coupling in order to design filters or mode couplers. Incorporating multiperiodic corrugations introduces new possibilities for filter applications. This is due to the fact that the multiperiodicity allows more resonant conditions to occur resulting in an intricate interaction picture. The problem is formulated in the format of the method of multiple scales, ${ }^{6}$ which is used to analyze the interaction of six propagating modes. Three incident modes and three reflected modes are coupled by both wall corrugations when three simultaneous resonant conditions occur; these are two resonant conditions on the codirectional modes and a Bragg condition. The coupledmode equations together with boundary conditions satisfied by the coupled modes constitute a two-point boundary value problem, which is solved numerically by the fundamental matrix method. ${ }^{7}$ The results are utilized for calculating the waveguide frequency response in terms of the power reflection coefficient. By introducing special types of nonuniformities such as amplitude taper and chirped corrugations, the filter characteristics can be modified to enhance certain desirable features.

\section{MATHEMATICAL MODEL}

As shown in Fig. 1, the periodic corrugations of the clad rod in a cylindrical coordinate system $(\hat{r}, \hat{\theta}, \hat{z})$ are given by $\hat{R}_{i}(\hat{z})=\hat{a}\left(1+\epsilon \cos \hat{k}_{w 1} \hat{z}\right)$, and $\hat{R}_{c}(\hat{z})=\hat{b}(1$ $\left.+\delta \epsilon \cos \hat{k}_{w 2} \hat{z}\right)$, where $\hat{a}$ and $\hat{b}$ are the average radii, $\hat{k}_{w 1}$ and $\hat{k}_{w 2}$ are, respectively, the wave numbers of the interface and the outerface, $\epsilon$ is a small dimensionless parameter much smaller than unity and equal to the ratio of the amplitude of the interface corrugation to $\hat{a}$, and $\delta$ is a constant allowing for a different corrugation amplitude at the outerface.

Assuming that both core and cladding are made of homogeneous, isotropic, and linearly elastic materials, then the governing equations of torsional motion are given as

$$
\frac{\partial^{2} \hat{u}_{\theta}}{\partial \hat{r}^{2}}+\frac{1}{\hat{r}} \frac{\partial \hat{u}_{\theta}}{\partial \hat{r}}-\frac{\hat{u}_{\theta}}{\hat{r}^{2}}+\frac{\partial^{2} \hat{u}_{\theta}}{\partial \hat{z}^{2}}-\frac{\hat{\rho}_{n}}{\hat{\mu}_{n}} \frac{\partial^{2} \hat{u}_{\theta}}{\partial \hat{t}^{2}}=0,
$$

where $\hat{u}_{\theta}$ is the $\theta$ component of displacement, $\hat{t}$ is the time coordinate, $\hat{\rho}_{n}$ are the densities, and $\hat{\mu}_{n}$ are the shear mod- 


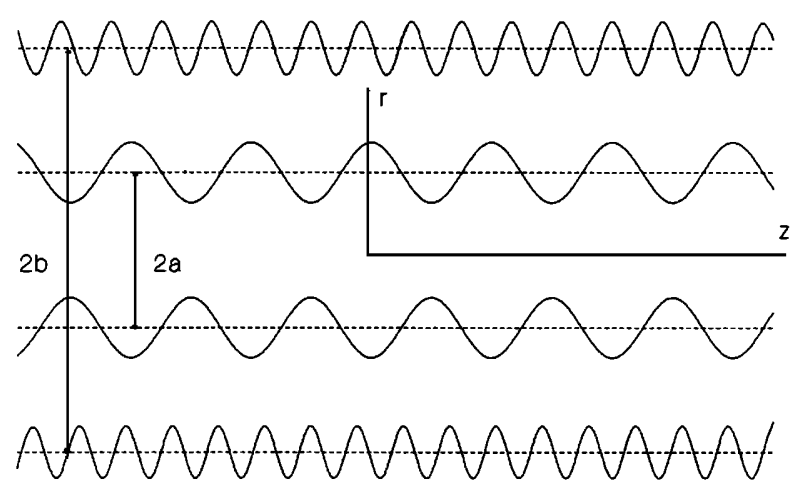

FIG. 1. Waveguide geometry.

uli of the two media. We consider propagation of monochromatic waves so that $\hat{u}_{\theta}$ in the core and the cladding are in the forms $\hat{u}_{\theta}=\hat{U}(\hat{r}, \hat{z}) \exp (-i \hat{\omega} \hat{t})$ and $\hat{u}_{\theta}=\hat{V}(\hat{r}, \hat{z})$ $\times \exp (-i \hat{\omega} \hat{t})$, respectively.

Moreover, we introduce dimensionless quantities without the carets by using $\hat{a}$ and $\hat{\omega}^{-1}$ as reference quantities. The following governing equations in dimensionless form are obtained:

$$
\begin{aligned}
& \frac{\partial^{2} U}{\partial r^{2}}+\frac{1}{r} \frac{\partial U}{\partial r}-\frac{U}{r^{2}}+\frac{\partial^{2} U}{\partial z^{2}}+k_{i}^{2} U=0, \\
& \frac{\partial^{2} V}{\partial r^{2}}+\frac{1}{r} \frac{\partial V}{\partial r}-\frac{V}{r^{2}}+\frac{\partial^{2} V}{\partial z^{2}}+k_{c}^{2} V=0,
\end{aligned}
$$

where $k_{i}=\hat{\omega} \hat{a}\left(\hat{\rho}_{i} / \hat{\mu}_{i}\right)^{1 / 2}$ and $k_{c}=\hat{\omega} \hat{a}\left(\hat{\rho}_{c} / \hat{\mu}_{c}\right)^{1 / 2}$ represent the wave numbers of bulk waves in the core and cladding materials, respectively.

The boundary conditions are the continuity of the displacement component $u_{\theta}$ and the stress vector $\bar{T}_{r \theta}$ at the interface $r=1+\epsilon \cos k_{w 1} z$, and the vanishing of $\bar{T}_{r \theta}$ at the outer boundary of the cladding $r=b+\epsilon \delta \cos k_{w 2} z$. Thus

$U=V, \quad$ at $\quad r=1+\epsilon \cos k_{w 1} z$,

$$
\begin{aligned}
& \mu\left[\left(\frac{\partial U}{\partial r}-\frac{U}{r}\right) n_{r}+\left(\frac{\partial U}{\partial z}\right) n_{z}\right] \\
& =\left(\frac{\partial V}{\partial r}-\frac{V}{r}\right) n_{r}+\left(\frac{\partial V}{\partial z}\right) n_{z}, \quad \text { at } \quad r=1+\epsilon \cos k_{w 1} z,
\end{aligned}
$$

$\left(\frac{\partial V}{\partial r}-\frac{V}{r}\right) n_{r}+\left(\frac{\partial V}{\partial z}\right) n_{z}=0, \quad$ at $\quad r=b+\epsilon \delta \cos k_{w 2} z$,

where $\mu=\hat{\mu}_{i} / \hat{\mu}_{c}$ and $n_{r}$ and $n_{z}$ are the components of the local outward-pointing unit normal.

\section{MULTIPLE SCALES FORMULATION}

We use the method of multiple scales ${ }^{6}$ and seek a firstorder perturbation expansions for $U$ and $V$ in powers of $\epsilon$ in the form

$$
\begin{aligned}
& U(r, z)=U_{0}\left(r, Z_{0}, Z_{1}, \ldots\right)+\epsilon U_{1}\left(r, Z_{0}, Z_{1}, \ldots\right)+\cdots, \\
& V(r, z)=V_{0}\left(r, Z_{0}, Z_{1}, \ldots\right)+\epsilon V_{1}\left(r, Z_{0}, Z_{1}, \cdots\right)+\cdots,
\end{aligned}
$$

where $Z_{0}=z$ is a short scale of the order of the wavelength in the guide and $Z_{1}=\epsilon z$ is a long scale that characterizes the amplitude and phase modulations due to the wall corrugations. Using the chain rule, we can write the derivatives with respect to $z$ in terms of $Z_{0}$ and $Z_{1}$ as

$$
\begin{aligned}
& \frac{\partial}{\partial z}=\frac{\partial}{\partial Z_{0}}+\epsilon \frac{\partial}{\partial Z_{1}}+\cdots, \\
& \frac{\partial^{2}}{\partial z^{2}}=\frac{\partial^{2}}{\partial Z_{0}^{2}}+2 \epsilon \frac{\partial^{2}}{\partial Z_{0} \partial Z_{1}}+\cdots .
\end{aligned}
$$

Substituting (7)-(10) into (2)-(6), expanding $U$ and $V$ at $r=1+\epsilon \cos k_{w 1} z$ in a Taylor series around $r=1, V$ at $r=b+\epsilon \delta \cos k_{w 2} z$ around $r=b$, and equating coefficients of equal powers of $\epsilon$ on both sides, we obtain

$O(1)$

$$
\begin{aligned}
& \frac{\partial^{2} U_{0}}{\partial r^{2}}+\frac{1}{r} \frac{\partial U_{0}}{\partial r}-\frac{U_{0}}{r^{2}}+\frac{\partial^{2} U_{0}}{\partial Z_{0}^{2}}+k_{i}^{2} U_{0}=0, \\
& \frac{\partial^{2} V_{0}}{\partial r^{2}}+\frac{1}{r} \frac{\partial V_{0}}{\partial r}-\frac{V_{0}}{r^{2}}+\frac{\partial^{2} V_{0}}{\partial Z_{0}^{2}}+k_{c}^{2} V_{0}=0, \\
& U_{0}-V_{0}=0, \quad \text { at } \quad r=1, \\
& \mu\left(\frac{\partial U_{0}}{\partial r}-U_{0}\right)-\frac{\partial V_{0}}{\partial r}+V_{0}=0, \quad \text { at } \quad r=1, \\
& \frac{\partial V_{0}}{\partial r}-\frac{V_{0}}{b}=0, \quad \text { at } \quad r=b .
\end{aligned}
$$

$O(\epsilon)$

$\frac{\partial^{2} U_{1}}{\partial r^{2}}+\frac{1}{r} \frac{\partial U_{1}}{\partial r}-\frac{U_{1}}{r^{2}}+\frac{\partial^{2} U_{1}}{\partial Z_{0}^{2}}+k_{i}^{2} U_{1}=-2 \frac{\partial^{2} U_{0}}{\partial Z_{0} \partial Z_{1}}$,

$\frac{\partial^{2} V_{1}}{\partial r^{2}}+\frac{1}{r} \frac{\partial V_{1}}{\partial r}-\frac{V_{1}}{r^{2}}+\frac{\partial^{2} V_{1}}{\partial Z_{0}^{2}}+k_{c}^{2} V_{1}=-2 \frac{\partial^{2} V_{0}}{\partial Z_{0} \partial Z_{1}}$,

$U_{1}-V_{1}=\cos k_{w 1} Z_{0}\left(\frac{\partial V_{0}}{\partial r}-\frac{\partial U_{0}}{\partial r}\right), \quad$ at $\quad r=1$,

$$
\begin{aligned}
\mu\left(\frac{\partial U_{1}}{\partial r}-U_{1}\right)-\frac{\partial V_{1}}{\partial r}+V_{1} \\
=-\cos k_{w 1} Z_{0}\left[\mu\left(\frac{\partial^{2} U_{0}}{\partial r^{2}}-\frac{\partial U_{0}}{\partial r}+U_{0}\right)\right. \\
\left.-\left(\frac{\partial^{2} V_{0}}{\partial r^{2}}-\frac{\partial V_{0}}{\partial r}+V_{0}\right)\right]-k_{w 1} \sin k_{w 1} Z_{0} \\
\quad \times\left[\mu\left(\frac{\partial U_{0}}{\partial Z_{0}}\right)-\frac{\partial V_{0}}{\partial Z_{0}}\right], \text { at } \quad r=1
\end{aligned}
$$

$$
\begin{aligned}
\frac{\partial V_{1}}{\partial r}-\frac{V_{1}}{b}= & -\delta \cos k_{w 2} Z_{0}\left(\frac{\partial^{2} V_{0}}{\partial r^{2}}-\frac{1}{b} \frac{\partial V_{0}}{\partial r}+\frac{V_{0}}{b^{2}}\right) \\
& -\delta k_{w 2} \sin k_{w 2} Z_{0}\left(\frac{\partial V_{0}}{\partial Z_{0}}\right), \text { at } \quad r=b .
\end{aligned}
$$




\section{SOLUTION OF THE REDUCED PROBLEM}

We seek solutions of (11) and (12) in the form of a linear combination of the six modes with wave numbers $k_{p}$, $k_{q}$, and $k_{s}$, that is,

$$
\begin{aligned}
U_{0}= & \sum_{j=p, q, s} J_{1}\left(\alpha_{j} r\right)\left[A_{j}^{+}\left(z_{1}\right) \exp \left(i k_{j} Z_{0}\right)\right. \\
& \left.+A_{j}^{-}\left(Z_{1}\right) \exp \left(-i k_{j} Z_{0}\right)\right], \\
V_{0}= & \sum_{j=p, q, s}\left[\Delta_{1 j} I_{1}\left(\gamma_{j} r\right)+\Delta_{2 j} K_{1}\left(\gamma_{j} r\right)\right] \\
& \times\left[A_{j}^{+}\left(Z_{1}\right) \exp \left(i k_{j} Z_{0}\right)+A_{j}^{-}\left(Z_{1}\right) \exp \left(-i k_{j} Z_{0}\right)\right],
\end{aligned}
$$

where the superscript "+" ("-") indicates incident (reflected) modes, $J_{1}$ is the Bessel function of the first kind, and $I_{1}$ and $K_{1}$ are the modified Bessel functions of the first and second kind, respectively. Here, $A_{j}^{\mp}\left(Z_{1}\right)$ are arbitrary functions determined from the solvability condition of the first-order problem, $\alpha_{j}^{2}=k_{i}^{2}-k_{j}^{2}, \quad \gamma_{j}^{2}=k_{j}^{2}-k_{c}^{2}, \quad \Delta_{1 j}$ $=J_{1}\left(\alpha_{j}\right) K_{2}\left(\gamma_{j} b\right) / \Delta_{j}$, and $\Delta_{2 j}=J_{1}\left(\alpha_{j}\right) I_{2}\left(\gamma_{j} b\right) / \Delta_{j}$, where $\Delta_{j}=I_{1}\left(\gamma_{j}\right) K_{2}\left(\gamma_{j} b\right)+K_{1}\left(\gamma_{j}\right) I_{2}\left(\gamma_{j} b\right)$.

Substitution of (21) and (22) into the boundary conditions given by (13)-(15) leads to the dispersion relation of guided modes

$$
\mu J_{2}\left(\alpha_{j}\right) \Delta_{j}-\left(\gamma_{j} / \alpha_{j}\right) J_{1}\left(\alpha_{j}\right) E_{j}=0
$$

where

$$
E_{j}=I_{2}\left(\gamma_{j} b\right) K_{2}\left(\gamma_{j}\right)-I_{2}\left(\gamma_{j}\right) K_{2}\left(\gamma_{j} b\right) .
$$

\section{SOLUTION OF THE FIRST-ORDER PROBLEM}

In the solution of the first-order approximation, one needs to distinguish between resonant and nonresonant cases; the latter case is that of decoupled modes, which is not discussed in this paper. In the resonant case, the modes are coupled and is the subject of this paper. The nearness of the resonances, which are introduced by the periodic interfaces, can be described by introducing the detuning parameters $\sigma_{1}, \sigma_{2}$, and $\sigma_{3}$ defined according to the following resonant conditions: ${ }^{8}$

$$
\begin{aligned}
& k_{p}-k_{q}=k_{w 1}+\epsilon \sigma_{1}, \\
& k_{p}-k_{s}=k_{w 2}+\epsilon \sigma_{2}, \\
& 2 k_{s}=k_{w 2}+\epsilon \sigma_{3} .
\end{aligned}
$$

Since the homogeneous first-order problem has a nontrivial solution, the inhomogeneous first-order problem has a solution if, and only if, a solvability condition is satisfied. ${ }^{6}$ To determine this condition, we seek a particular solution for $U_{1}$ and $V_{1}$ in the form

$$
\begin{aligned}
U_{1}= & \sum_{j=p, q, s}\left[\Lambda_{j}^{+}(r) \exp \left(i k_{j} Z_{0}\right)+\Lambda_{j}^{-}(r)\right. \\
& \left.\times \exp \left(-i k_{j} Z_{0}\right)\right],
\end{aligned}
$$

$$
\begin{aligned}
V_{1}= & \sum_{j=p, q, s}\left[\Gamma_{j}^{+}(r) \exp \left(i k_{j} Z_{0}\right)+\Gamma_{j}^{-}(r)\right. \\
& \left.\times \exp \left(-i k_{j} Z_{0}\right)\right] .
\end{aligned}
$$

Substituting (28) and (29), together with (21) and (22) into the governing equations (16) and (17), and equating the coefficients of $\exp \left(\mp i k_{j} Z_{0}\right)$ on both sides, we obtain

$$
\begin{aligned}
& {\left[\frac{d^{2}}{d r^{2}}+\frac{1}{r} \frac{d}{d r}+\left(\alpha_{j}^{2}-\frac{1}{r^{2}}\right)\right] \Lambda_{j}^{\mp}= \pm 2 i k_{j} \frac{d A_{j}^{\mp}}{d Z_{1}} J_{1}\left(\alpha_{j} r\right)} \\
& {\left[\frac{d^{2}}{d r^{2}}+\frac{1}{r} \frac{d}{d r}-\left(\gamma_{j}^{2}+\frac{1}{r^{2}}\right)\right] \Gamma_{j}^{\mp}} \\
& = \pm 2 i k_{j}\left(\frac{J_{1}\left(\alpha_{j}\right)}{\Delta_{j}}\right)\left[K_{2}\left(\gamma_{j} b\right) I_{1}\left(\gamma_{j} r\right)\right. \\
& \left.+I_{2}\left(\gamma_{j} b\right) K_{1}\left(\gamma_{j} r\right)\right] \frac{d A_{j}^{\mp}}{d Z_{1}}
\end{aligned}
$$

where $j=p, q, s$.

We multiply (30) by $r J_{1}\left(\alpha_{j} r\right)$ and integrate by parts from $r=0$ to $r=1$. Similarly, we multiply (31) first by $r I_{1}\left(\gamma_{j} r\right)$ and integrate by parts from $r=1$ to $r=b$, then by $r K_{1}\left(\gamma_{j} r\right)$ and integrate by parts from $r=1$ to $r=b$. This leads to the following equations:

$$
\begin{aligned}
& \Lambda_{j}^{\mp \prime}(1) J_{1}\left(\alpha_{j}\right)-\Lambda_{j}^{\mp}(1)\left[J_{1}\left(\alpha_{j}\right)-\alpha_{j} J_{2}\left(\alpha_{j}\right)\right] \\
& \quad=\mp i \Phi_{1 j} \frac{d A_{j}^{\mp}}{d Z_{1}}, \\
& b I_{1}\left(\gamma_{j} b\right) \Gamma_{j}^{\mp \prime}(b)-I_{1}\left(\gamma_{j}\right) \Gamma_{j}^{\mp \prime}(1)-b \gamma_{j} I_{1}^{\prime}\left(\gamma_{j} b\right) \Gamma_{j}^{\mp}(b) \\
& \quad+\gamma_{j} I_{1}^{\prime}\left(\gamma_{j}\right) \Gamma_{j}^{\mp}(1)=\mp i \Phi_{2 j} \frac{d A_{j}^{\mp}}{d Z_{1}}, \\
& b K_{1}\left(\gamma_{j} b\right) \Gamma_{j}^{\mp \prime}(b)-K_{1}\left(\gamma_{j}\right) \Gamma_{j}^{\mp \prime}(1)-b \gamma_{j} K_{1}^{\prime}\left(\gamma_{j} b\right) \Gamma_{j}^{\mp}(b) \\
& \quad+\gamma_{j} K_{1}^{\prime}\left(\gamma_{j}\right) \Gamma_{j}^{\mp}(1)=\mp i \Phi_{3 j} \frac{d A_{i}^{\mp}}{d Z_{1}},
\end{aligned}
$$

where primes indicate derivatives with respect to the arguments, and the constants $\Phi_{1 j}, \Phi_{2 j}$, and $\Phi_{3 j}$ are given in the Appendix.

The values of the functions $\Lambda_{j}, \Gamma_{j}$, and their derivatives evaluated at the interfaces and appearing on the lefthand side of Eqs. (32)-(34) are determined from the boundary conditions in the manner outlined by Asfar and Nayfeh. ${ }^{5}$ This leads to the following coupled mode equations:

$$
\begin{aligned}
& C_{p p} \frac{d A_{p}^{+}}{d Z_{1}}+C_{p q} A_{q}^{+} e^{-i \sigma_{1} Z_{1}}+C_{p s} A_{s}^{+} e^{-i \sigma_{2} Z_{1}}=0, \\
& C_{q q} \frac{d A_{q}^{+}}{d Z_{1}}+C_{q p} A_{p}^{+} e^{i \sigma_{1} Z_{1}}=0 \\
& C_{s s} \frac{d A_{s}^{+}}{d Z_{1}}+C_{s p} A_{p}^{+} e^{i \sigma_{2} Z_{1}}+C_{s s n} A_{s}^{-} e^{-i \sigma_{3} Z_{1}}=0
\end{aligned}
$$




$$
\begin{aligned}
& -C_{p p} \frac{d A_{p}^{-}}{d Z_{1}}+C_{p q} A_{q}^{-} e^{i \sigma_{1} Z_{1}}+C_{p s} A_{s}^{-} e^{i \sigma_{2} Z_{1}}=0, \\
& -C_{q q} \frac{d A_{q}^{-}}{d Z_{1}}+C_{q p} A_{p}^{-} e^{-i \sigma_{1} Z_{1}}=0, \\
& -C_{s s} \frac{d A_{s}^{-}}{d Z_{1}}+C_{s p} A_{p}^{-} e^{-i \sigma_{2} Z_{1}}+C_{s s n} A_{s}^{+} e^{i \sigma_{3} Z_{1}}=0,
\end{aligned}
$$

where $C_{j j}, C_{p q}, C_{p s}, C_{q p}, C_{s p}$, and $C_{s s n}$ are given in the Appendix.

\section{FILTER DESIGN EXAMPLES}

The system of equations (35)-(40) may be written in the matrix form

$$
\left\{y^{\prime}\right\}=[P]\{y\},
$$

where $\{y\}$ is a six-vector whose components are the mode amplitudes and $[P]$ is a $6 \times 6$ matrix of the coupling coefficients. The boundary conditions on both ends of the filter section may be written in the form

$$
\begin{aligned}
& {[Q]\{y(O)\}=\left\{w_{0}\right\},} \\
& {[R]\{y(L)\}=\{0\},}
\end{aligned}
$$

where $[Q]$ and $[R]$ are each a $3 \times 6$ constant matrix, $\left\{w_{0}\right\}$ is a $3 \times 1$ column vector representing the excitation amplitudes of the incident modes, and the right-hand side of (43) expresses the fact that the reflected modes vanish at the end of the corrugated section.

The problem defined by (41)-(43) constitutes a twopoint boundary value problem. Such problems can be solved numerically by an efficient code based on the fundamental matrix method. ${ }^{7}$ The missing boundary conditions are found, and consequently, the power reflection coefficient can be calculated.

For the purpose of illustration, a composite rod with $b=1.1, \rho_{c} / \rho_{i}=3$, and $\mu_{c} / \mu_{i}=50$ is selected. The necessary condition for resonance $\left(k_{p} \approx 3 k_{s}\right)$ is found to be at $\omega=845300 \mathrm{~Hz}$. The wall wave numbers were accordingly chosen as $k_{w 1}=1.920$, and $k_{w 2}=6.606$. The length of the corrugated section $L=10$, and the perturbation parameter $\epsilon=0.1$.

The characteristic determinant of the system (41) $(\operatorname{det}[P]-\lambda[I=0)$ is a polynomial of the sixth degree whose roots are the eigenvalues of the system. The level of attenuation is higher in the frequency range which has more complex eigenvalues. For filter design, it is necessary that all six eigenvalues be complex in the frequency band around resonance. This requirement has been found difficult to realize when the two periodic corrugations are placed at separate interfaces with no more than two complex eigenvalues instead of six even when the resonant conditions (25)-(27) are satisfied (Bragg condition on the outerface). The interaction can be strengthened by exchanging the resonance conditions between the interface and the outerface; i.e., $k_{p}-k_{q}=k_{w 2}+\epsilon \sigma_{1}, k_{p}-k_{s}=k_{w 1}$ $+\epsilon \sigma_{2}$, and $2 k_{s}=k_{w 1}+\epsilon \sigma_{3}$. This makes four of the eigenvalues complex around resonance. This effect is due to the

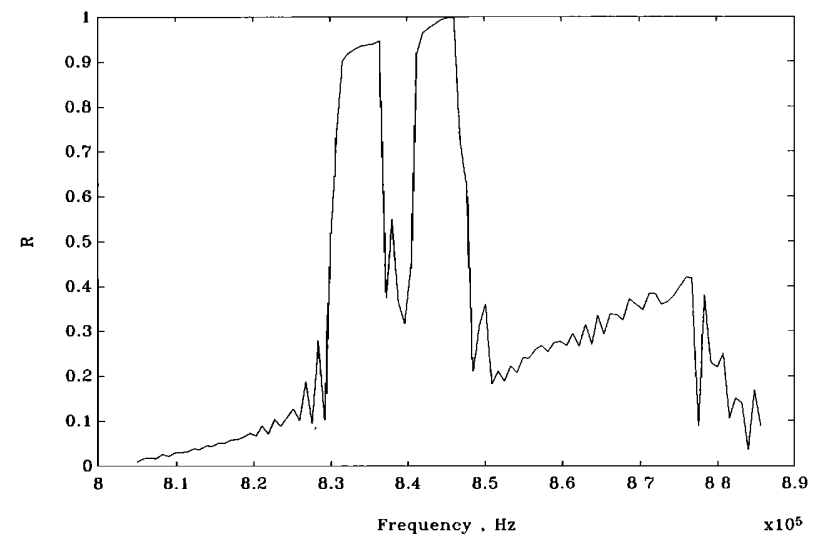

FIG. 2. Power reflection coefficient $R$ for a waveguide with uniform corrugations.

fact that the corrugations on the outerface couple the Bragg modes weakly because the acoustic field is weak there. This is mainly because the modified Bessel functions decay sharply in the clad away from the interface. These observations motivate bringing the two periodicities together at the clad-rod interface thus leaving the outerface smooth. Numerical investigation of the latter case shows that all eigenvalues are complex in the frequency band around resonance. This choice of preiodic corrugation is therefore used as a basis for the filter design examples discussed in the sequel.

The power reflection coefficient versus frequency for the case of uniform corrugations is depicted in Fig. 2. The figure shows a typical filter response with nearly total reflection at midband, and side ripples whose level decreases as one moves away from the resonant frequency. In order to enhance the filter characteristics, two types of nonuniform corrugations are used. First, a raised sinusoidal taper of the form $\epsilon\left(Z_{1}\right)=\epsilon_{0}\left[\sin \left(k_{w 1} Z_{1} / L^{2}\right)\right]^{1 / 3}$ is applied to both wall distortion functions. Figure 3 shows the effect of amplitude tapering in giving a better rolloff characteristic, and shows "clean" sides due to the disappearance of side ripples. However, the response is still far from ideal. When, a linear chirp of the form $k_{w 1}\left(Z_{1}\right)=1.920+F\left(Z_{1}-L / 2\right) /$

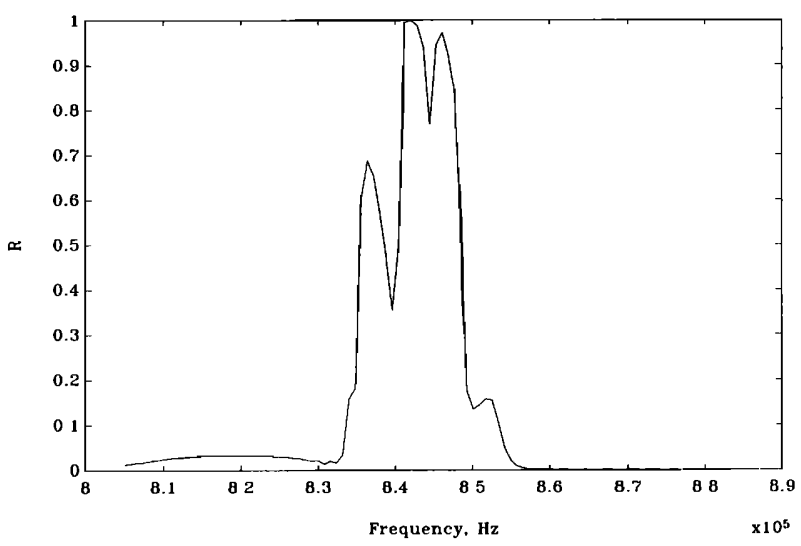

FIG. 3. Power reflection coefficient $R$ for a waveguide with tapered corrugations, $\epsilon_{0}=0.1$. 


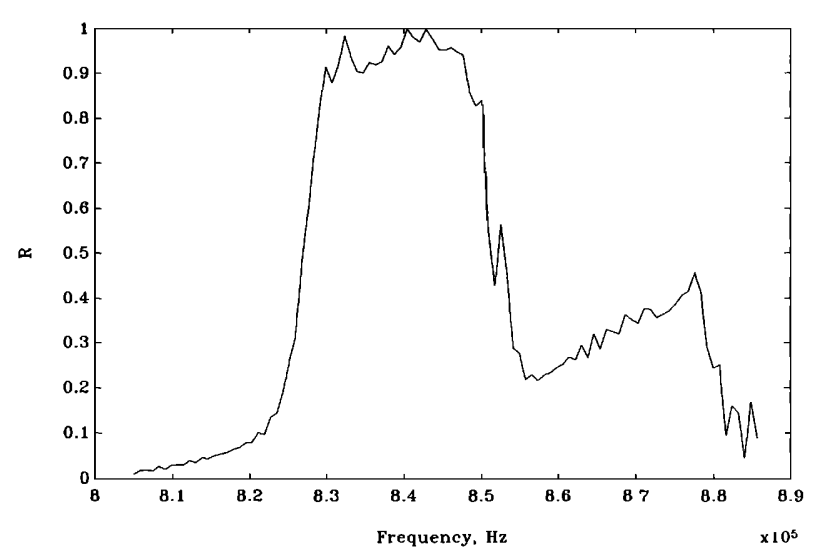

FIG. 4. Power reflection coefficient $R$ for a waveguide with chirped corrugations, $F=1.0$.

$L^{2}$ is imposed on the first distortion function, and $k_{w 2}\left(Z_{1}\right)$ $=6.606-F\left(Z_{1}-L / 2\right) / L^{2}$ on the second distortion function, a wide midband response is obtained as can be seen from Fig. 4. However, the presence of the side ripples is not desirable. An optimal filter may be realized by a combination of taper and chirp whereby the side ripples are suppressed resulting in a narrow band response as obvious from Fig. 5.

\section{CONCLUSION}

We have investigated the interaction of six torsional elastic modes in a composite waveguide having multiperiodic interface corrugations. The resonant conditions under which the interaction took place were found to be of critical importance in designing the waveguide as a filter. A reasonable choice was to apply two periodicities on the clad-rod interface. Further trials to achieve better filteration characteristics were done by imposing different types of nonuniformities on the corrugations, including taper and chirped corrugations. Certain desirable features were realized, however some shortcomings remained. Therefore a combination of taper and chirp was finally adopted in order to optimize the design.

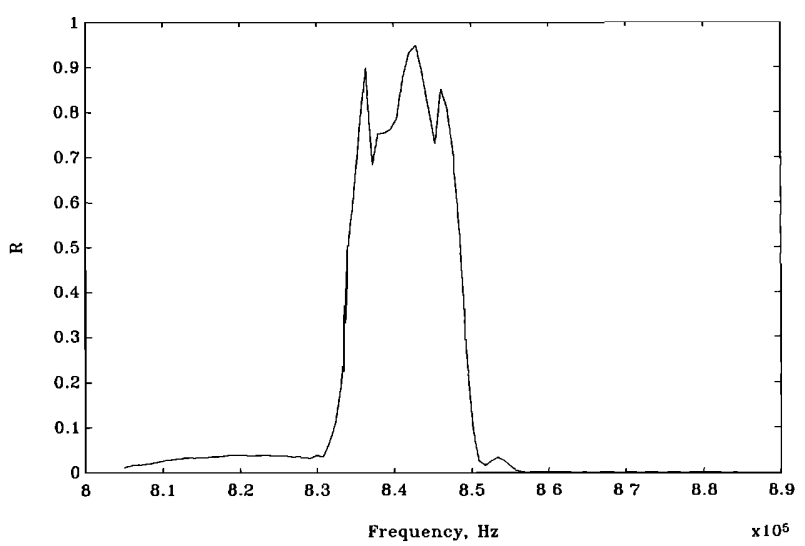

FIG. 5. Power reflection coefficient $R$ for a waveguide with a combination of tapered and chirped corrugations.

\section{APPENDIX}

$$
\begin{aligned}
\Phi_{1 j}= & -k_{j}\left[J_{1}^{2}\left(\alpha_{j}\right)-J_{0}\left(\alpha_{j}\right) J_{2}\left(\alpha_{j}\right)\right], \\
\Phi_{2 j}= & -k_{j}\left[J_{1}\left(\alpha_{j}\right) / \Delta_{j}\right]\left\{0.5 M_{j} I_{2}\left(\gamma_{j} b\right)+K_{2}\left(\gamma_{j} b\right)\right. \\
& \times\left[b^{2}\left(I_{1}^{2}\left(\gamma_{j} b\right)-I_{0}\left(\gamma_{j} b\right) I_{2}\left(\gamma_{j} b\right)\right)\right] \\
& \left.-\left[\left(I_{1}^{2}\left(\gamma_{j}\right)-I_{0}\left(\gamma_{j}\right) I_{2}\left(\gamma_{j}\right)\right)\right]\right\}, \\
\Phi_{3 j}= & -k_{j}\left[J_{1}\left(\alpha_{j}\right) / \Delta_{j}\right]\left\{0.5 M_{j} K_{2}\left(\gamma_{j} b\right)+I_{2}\left(\gamma_{j} b\right)\right. \\
& \times\left[b^{2}\left(K_{1}^{2}\left(\gamma_{j} b\right)-K_{0}\left(\gamma_{j} b\right) K_{2}\left(\gamma_{j} b\right)\right)\right] \\
& \left.-\left[\left(K_{1}^{2}\left(\gamma_{j}\right)-K_{0}\left(\gamma_{j}\right) K_{2}\left(\gamma_{j}\right)\right)\right]\right\},
\end{aligned}
$$

where

$$
\begin{aligned}
M_{j}= & b^{2}\left[2 I_{1}\left(\gamma_{j} b\right) K_{1}\left(\gamma_{j} b\right)-I_{0}\left(\gamma_{j} b\right) K_{2}\left(\gamma_{j} b\right)\right. \\
& \left.-I_{2}\left(\gamma_{j} b\right) K_{0}\left(\gamma_{j} b\right)\right]-\left[2 I_{1}\left(\gamma_{j}\right) K_{1}\left(\gamma_{j}\right)\right. \\
& \left.-I_{0}\left(\gamma_{j}\right) K_{2}\left(\gamma_{j}\right)-I_{2}\left(\gamma_{j}\right) K_{0}\left(\gamma_{j}\right)\right], \\
C_{j j}= & i\left[\Phi_{1 j} \xi_{1 j}-\Phi_{2 j} \xi_{2 j}+\Phi_{3 j} \xi_{3 j}\right], \\
C_{p q}= & -\Psi_{3 p}\left(h_{q}+h_{q}^{*}\right), \\
C_{p s}= & \Psi_{1 p} f_{s}+\Psi_{2 p}\left(g_{s}+g_{s}^{*}\right), \\
C_{q p}= & -\Psi_{3 q}\left(h_{p}-h_{p}^{*}\right), \\
C_{s p}= & \Psi_{1 s} f_{p}+\Psi_{2 s}\left(g_{p}-g_{p}^{*}\right), \\
C_{s s n}= & \Psi_{1 s} f_{s}+\Psi_{2 s}\left(g_{s}-g_{s}^{*}\right),
\end{aligned}
$$

where

$$
\begin{aligned}
f_{j}= & -\frac{1}{2}\left[\left(\gamma_{j} J_{1}\left(\alpha_{j}\right) / \Delta_{j}\right) E_{j}+J_{1}\left(\alpha_{j}\right)-\alpha_{j} J_{2}\left(\alpha_{j}\right)\right], \\
g_{j}= & \frac{1}{2}\left\{\left(J_{1}\left(\alpha_{j}\right) / \Delta_{j}\right)\left[\gamma_{j}^{2} \eta_{j}-2 \gamma_{j} E_{j}\right]-\mu\left[\alpha_{j}^{2} J_{3}\left(\alpha_{j}\right)\right.\right. \\
& \left.\left.\quad-2 \alpha_{j} J_{2}\left(\alpha_{j}\right)\right]\right\} \\
g_{j}^{*}=\frac{1}{2} k_{w 1} k_{j} J_{1}\left(\alpha_{j}\right)(1-\mu), & \\
h_{j}= & -\frac{1}{2} \delta\left(J_{1}\left(\alpha_{j}\right) / \Delta_{j}\right) \gamma_{j}^{2} L_{j}, \\
h_{j}^{*}= & -\frac{1}{2} \delta k_{w 2} k_{j}\left(J_{1}\left(\alpha_{j}\right) / \Delta_{j}\right) N_{j},
\end{aligned}
$$

and

$$
\begin{aligned}
\eta_{j}= & I_{3}\left(\gamma_{j}\right) K_{2}\left(\gamma_{j} b\right)+K_{3}\left(\gamma_{j}\right) I_{2}\left(\gamma_{j} b\right), \\
L_{j}= & I_{3}\left(\gamma_{j} b\right) K_{2}\left(\gamma_{j} b\right)+K_{3}\left(\gamma_{j} b\right) I_{2}\left(\gamma_{j} b\right), \\
N_{j}= & I_{1}\left(\gamma_{j} b\right) K_{2}\left(\gamma_{j} b\right)+K_{1}\left(\gamma_{j} b\right) I_{2}\left(\gamma_{j} b\right), \\
\Psi_{v j}= & \beta_{1 v j} \xi_{1 j}-\beta_{2 v j} \xi_{2 j}+\beta_{3 v j} \xi_{3 j}, \quad v=1,2,3, \\
\xi_{1 j}= & \left(\beta_{22 j}-I_{1}\left(\gamma_{j}\right)\right)\left(b K_{1}\left(\gamma_{j} b\right)-\beta_{33 j}\right) \\
& \quad-\left(\beta_{32 j}-K_{1}\left(\gamma_{j}\right)\right)\left(b I_{1}\left(\gamma_{j} b\right)-\beta_{23 j}\right), \\
\xi_{2 j}= & \beta_{12 j}\left(b I_{1}\left(\gamma_{j} b\right)-\beta_{23 j}\right), \\
\xi_{3 j}= & \beta_{12 j}\left(b K_{1}\left(\gamma_{j} b\right)-\beta_{33 j}\right),
\end{aligned}
$$




$$
[\beta]=\frac{1}{1-\mu}\left[\begin{array}{ccc}
\alpha_{j} J_{2}\left(\alpha_{j}\right)-J_{1}\left(\alpha_{j}\right) & \alpha_{j} J_{2}\left(\alpha_{j}\right)-J_{1}\left(\alpha_{j}\right) & 0 \\
\mu \gamma_{j} I_{1}^{\prime}\left(\gamma_{j}\right) & \gamma_{j} I_{1}^{\prime}\left(\gamma_{j}\right) & b^{2} \gamma_{j}(1-\mu) I_{1}^{\prime}\left(\gamma_{j} b\right) \\
\mu \gamma_{j} K_{1}^{\prime}\left(\gamma_{j}\right) & \gamma_{j} K_{1}^{\prime}\left(\gamma_{j}\right) & b^{2} \gamma_{j}(1-\mu) K_{1}^{\prime}\left(\gamma_{j} b\right)
\end{array}\right],
$$

where prime indicates a derivative with respect to $r$.

${ }^{1}$ A. H. Meitzler, J. Acoust. Soc. Am. 33, 435-445 (1961).

${ }^{2}$ M. Hirao, J. Acoust. Soc. Am. 67, 1-5 (1980).

${ }^{3}$ J. D. Kim and H. H. Bau, J. Appl. Mech. 58, 710-715 (1991).

${ }^{4}$ O. R. Asfar and A. H. Nayfeh, IEEE Trans. Microwave Theory Tech. MTT-23, 728-734 (1975).
${ }^{5}$ O. R. Asfar and A. H. Nayfeh, IEEE Trans. Sonics Ultrason. SU24,313-317 (1977).

${ }^{6}$ A. H. Nayfeh, Introduction to Perturbation Techniques (Wiley, New York, 1981).

${ }^{7}$ O. R. Asfar and A. M. Hussein, Int. J. Num. Meth. Eng. 28, 1205-1216 (1989).

${ }^{8}$ O. R. Asfar, J. Elec. Waves Appl. 3, 697-709 (1989). 